\title{
Un acercamiento cuantitativo a los titulares de la prensa después de la primavera árabe
}

\section{A quantitative approach to headlines after the Arab Spring}

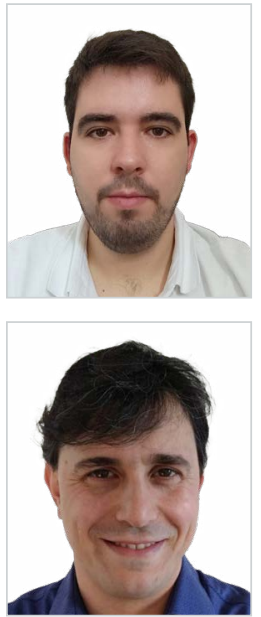

Recibido: 17/05/2021- Aceptado: 30/09/2021 - En edición: 22/10/2021 - Publicado: 01/12/2021

\section{Resumen:}

Las implicaciones y consecuencias de la Primavera Árabe no pasaron inadvertidas para los medios de comunicación de todo el mundo. En primer lugar, porque las revueltas supusieron uno de los acontecimientos más inesperados de la pasada década. Y, en segundo lugar, porque los medios de comunicación, especialmente las redes sociales, pudieron jugar un papel fundamental en las protestas. Más de nueve años después, hay pocos elementos para hablar de éxito en cuanto a la democratización de los países que experimentaron estas movilizaciones populares. Ante esta realidad se resalta la cuestión sobre qué ha quedado de la Primavera Árabe en los medios de comunicación españoles. La actual investigación explora los principales temas tra-
Received: 17/05/2021 - Accepted: 30/09/2021 - Early access: 22/10/2021 - Published: 01/12/2021 Abstract:

The implications and consequences of the Arab Spring did not go unnoticed by the media around the world. Firstly, because the riots were one of the most unexpected events of the last decade. And secondly, because the media, especially social media, could play a key role in the protests. More than nine years later, there are few elements to speak of success in terms of the democratization of the countries that experienced these popular mobilizations. Faced with this reality, the question of what has remained of the Arab Spring in the Spanish media is highlighted. The current research explores the main topics covered by the Spanish press during the post-Arab Spring period (2012-2020). The Latent Dirichlet Allocation (LDA) algorithm was used to do this. The results show that

Cómo citar este artículo:

Moreno Mercado, J. M. y García Marín, J. (2021). Un acercamiento cuantitativo a los titulares de la prensa después de la primavera árabe. Doxa Comunicación, 33, pp. 347-362. 
tados por la prensa española durante el periodo post-Primavera Árabe (2012-2020). Para ello se ha utilizado el algoritmo Latent Dirichlet Allocation (LDA). Los resultados muestran que la prensa española ha centrado su atención, especialmente, en elementos conflictivos de los países que experimentaron revueltas. Además, se observó una sobredimensión informativa de las realidades de los países árabes culturalmente cercanos. El objetivo no es solo realizar una aproximación teórica sobre el objeto de estudio sino reforzar la utilidad de esta técnica en trabajos propios de comunicación política.

\section{Palabras clave:}

Medios de comunicación; primavera árabe; prensa; titulares; LDA. the Spanish press has focused its attention, especially, on conflicting elements in the countries that experienced revolts. In addition, there was an informative over-dimensioning of the realities of culturally close Arab countries. The objective is not only to make a theoretical approach on the object of study but to reinforce the usefulness of this tecÝique in its own works of political communication.

Keywords:

Mass media; Arab Spring; press; headlines; LDA.

\section{Introducción}

La Primavera Árabe fue uno de los episodios políticos más impredecibles de los últimos tiempos (Atlas, 2012). Ante todo, por desarrollarse en una región caracterizada por un control férreo de la información con una libertad de prensa y expresión ampliamente coartada (García-Marín, 2017). Y, a continuación, porque invalidó las tesis que afirmaban que era altamente improbable que las sociedades árabes fueran el epicentro de grandes movilizaciones sociales. En otras palabras, postulados culturalistas que hablaban de la excepcionalidad islámica (Abu-Tarbush, 2015: 31). Por supuesto, las protestas que se iniciaron a finales del año 2010 han dado lugar a una enorme cantidad de literatura académica de diversas disciplinas, entre las cuales, evidentemente, no falta la ciencia política y, concretamente, la comunicación política (Durán-Cenit y García-Marín, 2015; Altourah, Chen y Al-Kandari, 2021).

Uno de los elementos más comentados de la Primavera Árabe fue el papel que jugaron los medios de comunicación y, sobre todo, las redes sociales (Meraz y Papacharissi, 2013). La autoinmolación de Mohammed Bouazizi en Túnez no fue la primera, ni la única, del año pero sí la que desencadenó las movilizaciones que derrocaron al régimen de Ben Ali y que, posteriormente, se expandieron por toda la región. La explicación quizá la encontramos en que la información se dirigió a la prensa internacional y no a los ciudadanos (Martínez-Fuentes, 2011) conjugándose así dos sucesos extraordinarios, redes sociales (internas) y medios de comunicación (externos). Este hecho resulta realmente importante si tenemos en cuenta que muchos de los periodistas internacionales tenían prohibida la entrada al país magrebí. Prohibición que también se dio en otros países, como en Siria al comienzo de la revolución.

En la actualidad, la zona sigue contando con puntuaciones muy restrictivas en materia de derechos sociales y libertades civiles, exceptuando el caso tunecino (Freedom House, 2020; The Economist, 2019); cerrando aquellos debates que especulaban que pudiera darse una cuarta ola de democratización (Priego-Moreno, 2011). Aunque, es cierto que los efectos de la Primavera Árabe han afectado a prácticamente todos los países del mundo arabo-musulmán. Efectos que sí pueden encuadrarse dentro de una ola de cambio político (Szmolka, 2013). Por supuesto, dentro del debate académico en torno a las lecciones aprendidas de dichas revueltas debe incluirse el papel de los medios de comunicación, objeto de estudio de la presente investigación. 
Este trabajo intenta estudiar cómo se han construido los titulares de la prensa española, concretamente en los casos de El País, ABC, El Mundo y La Vanguardia, durante el periodo post-Primavera Árabe (2012-2020). Para ello se han analizado los titulares de prensa de cada medio explorando la frecuencia de publicación de noticias, la importancia que se le da a los distintos países y a los encuadres principales a la hora de informar sobre la región. A juicio de los investigadores, el análisis de titulares se muestra pertinente ya que, si bien, pueden no reflejar íntegramente el contenido de la noticia, la necesidad de que los usuarios cliqueen en los enlaces ha favorecido el uso de encabezamientos atractivos e impactantes (el llamado popularmente clickbait). Para ello, se utilizará el algoritmo no-supervisado LDA (Latent Dirichlet Allocation), muy útil para resumir volúmenes amplios de datos con textos uniformes.

El presente trabajo queda estructurado de la siguiente manera. Tras la presente introducción se hará un repaso teórico sobre la importancia de los medios de comunicación durante el desarrollo de la Primavera Árabe. Posteriormente, se explicará el enfoque metodológico utilizado y los principales resultados de su aplicación. Cierra la investigación, un último apartado dedicado a extraer las conclusiones más relevantes.

\section{La primavera árabe y los medios de comunicación}

La Primavera Árabe o Despertar Árabe ha sido uno de los acontecimientos de cambio político más relevantes de lo que llevamos del siglo XXI. Nueve años después del derrocamiento de Ben Ali en Túnez, Muammar el Gaddafi en Libia, Hosni Mubarak en Egipto y Abdullah Saleh en Yemen existen pocos elementos que puedan afirmar que se ha producido una democratización de la región (Álvarez-Ossorio, Barreñada-Bajo y Mijares-Molina, 2021). Incluso, en los últimos años las protestas masivas en otros países, concretamente Argelia, Sudán, Líbano e Irak donde las primeras movilizaciones quedaron en segundo plano, han propiciado el debate acerca de si todavía existe una continuación de la Primavera Árabe o simplemente un nuevo desarrollo político regional (Truevtsev, 2020). A pesar de que los principales índices sobre democracia y libertades arrojan resultados escépticos en esta cuestión, autores como El-Haddad (2020) afirman que uno de los efectos de la Primavera Árabe ha sido la reconfiguración del contrato social entre sociedad civil y élites políticas.

Uno de los aspectos más señalados a la hora de abordar las movilizaciones en el mundo árabe ha sido el uso de los medios de comunicación y las redes sociales. No obstante, hay que advertir que no existe una respuesta unánime acerca de su importancia. En otras palabras, en cada país los medios de comunicación adoptaron un papel diferente aunque interrelacionado. Por ejemplo, en el caso de Túnez las redes sociales sirvieron como un canal de información exterior, siendo Al Jazeera la primera cadena en mostrar casi todos los vídeos que provenían del país (Sarnelli, 2013). Mientras, que, en el caso de Egipto, las redes sociales sirvieron como un factor organizador y de coordinación, no solo de información (Wilson y Dunn, 2011). Sin embargo, diversos autores señalan que se ha mitificado la importancia de los medios y las redes sociales como vía democratizadora. En palabras de Moldovan (2020): “Las secuelas de la Primavera Árabe demostraron que un cambio político significativo en la región MENA no puede ser provocado solo por la movilización a través de las redes sociales y las protestas televisadas” (p. 268). Haciendo alusión

1 Acrónimo de Middle East and North Africa. 
a que hacen falta otros factores, como una correcta cultura política democrática, controles a la separación de poderes, entre otros muchos.

Más allá del debate sobre los posibles efectos que pudieron condicionar el desarrollo de las protestas, un asunto que ha preocupado a académicos de todo el mundo ha sido qué imagen trasladaron los medios de comunicación sobre estas. De ahí que hallemos una considerable cantidad de trabajos sobre diversas cuestiones con resultados significativos. Por ejemplo, Dastgeer y Gade (2016) señalan cómo las mujeres tuvieron un papel protagonista en la cobertura de medios como Al Jazeera, a pesar de que los medios árabes poseen una clara orientación masculina (Engineer, 2008). Mientras que, Sik Ha y Shin (2019) indican que los medios chinos minimizaron los relatos de los ciudadanos y los activistas sociales acusando a las naciones occidentales de imponer un concepto interesado de orden y seguridad.

Las revueltas en el mundo árabe tampoco pasaron inadvertidas para los medios de comunicación españoles, aunque dicha cobertura no ha estado exenta de críticas. Un elemento a destacar fue la tardía cobertura de los sucesos acaecidos en Túnez, epicentro de las protestas. Corral-García y Fernández-Romero (2015) apuntan que los principales diarios de prensa españoles tardaron más de un mes, coincidiendo con la caída de Ben Ali, en publicar en sus portadas las movilizaciones. Y, en el caso de TV3, en Cataluña, el uso de medios sociales como fuente de información fue prácticamente inexistente (Elena y Tulloch, 2017) cuando reportaron las protestas contra el régimen de Mubarak.

En general, se puede afirmar que la prensa española vio de una manera positiva la caída de los regímenes dictatoriales (Sanz-Ocaña, 2018) aunque Irakrak (2017) añade que dicha imagen estuvo condicionada a conceptos preestablecidos sobre la sociedad y cultura árabe. Imágenes preestablecidas que en algunos casos, trascienden más allá de la Primavera Árabe, véase el caso de Arabia Saudí (Omar Alsalem, 2016). Empero, las derivas posteriores en algunos países, como la guerra en Libia tras la caída de Gaddafi o la victoria de los Hermanos Musulmanes en Egipto, ha rescatado la antigua imagen pesimista que los medios españoles proyectaban de los países árabes (Vidal-Valiña, 2013). En otras palabras, los diarios españoles apoyaron a los sectores populares cuando estos se movilizaron contra los regímenes establecidos, véase el caso de Libia, (Soria-Ibáñez, 2013), pero encuadraron los conflictos desde otros aspectos (como la seguridad), cuando estos se prolongaron, véase el caso de Siria (Moreno-Mercado, 2018).

Si realizamos un repaso de la literatura más reciente sobre el objeto de estudio, se puede inferir que las investigaciones se centran más en las consecuencias de las revueltas que los fundamentos que llegaron a motivarlas. Entre esas consecuencias destacamos la aparición de discursos islamófobos que señalan la incompatibilidad de la democracia con el islam como factor explicativo (Corral, Fernández-Romero, García-Ortega, 2020), los encuadres utilizados ante la cuestión de los refugiados (Sánchez-Castillo y Zarauza-Valero, 2020) o el interés informativo sobre grupos terroristas que operan en países del mundo árabe (Plaza, Rivas-Nieto y Rey García, 2017), entre otras.

\section{Metodología}

El presente estudio se ha basado en un análisis cuantitativo, sin abandonar elementos cualitativos, de la prensa española; en concreto en cuatro diarios de tirada nacional, El País, El Mundo, ABC y La Vanguardia en su versión digital. Dicha versión nos permite contar con un número de unidades de análisis importante y adaptarse más a la realidad digital de la sociedad española 
(Luengo y Fernández-García, 2017). El periodo de estudio comprende 8 años, concretamente de 2012 a 2020, tomando como referencia de inicio el año posterior a las revueltas de 2011. La selección de noticias se ha extraído de la base de datos de MyNews lo que ha permitido recopilar una muestra considerable $(n=4.629)$ que permita reflejar una imagen fiel de la cobertura española durante el periodo post-Primavera Árabe. A pesar de que esta investigación tiene como objetivo analizar los titulares, se han extraído las noticias que en el contenido aparecieran los siguientes términos: *Primavera y *Árabe. La razón es no limitar en exceso la muestra.

El estudio de los titulares de prensa ha sido un elemento recurrente en las investigaciones sobre comunicación política. Según Van Dijk (1990), estos representan una imagen concisa y resumida de la noticia y define la orientación del medio ante la cuestión tratada. A nuestro juicio, dicho estudio se muestra muy pertinente ya que las lógicas informativas actuales apuntan a que la necesidad de obtener visitas y clics, ha obligado a los medios de comunicación a elaborar titulares más atractivos para las audiencias, un proceso que alcanza a casi todos los medios europeos (Orosa, Santorum y García, 2017). En otras palabras, el elemento emotivo se está sobreponiendo al factor informativo.

La extracción de temas a partir de los titulares ha sido posible gracias a la automatización de análisis de texto a partir del algoritmo no-supervisado LDA (Latent Dirichlet Allocation). LDA es probablemente el modelo probabilístico generativo más utilizado de los llamados Topic Models (Hovy, 2020). Antes de aplicar el algoritmo los textos han sido preprocesados (tokenizados) eliminando elementos que pueden alterar el análisis. Entre ellos, espacios en blanco, puntuaciones, preposiciones y palabras comunes (stopwords). El algoritmo LDA permite agrupar los titulares en determinados clústeres a partir de la asociación de palabras clave que representan el tema principal de cada grupo. El modelo parte de la hipótesis de que la persona que escribe un documento posee ciertos temas claves en la mente (Ostrowiski, 2015). Este gráficamente se plasma en una serie de cajas introducidas unas sobre las otras. "Las cajas son " "placas"' que representan réplicas. La placa exterior representa los documentos, mientras que la placa interior representa la elección repetida de temas y palabras dentro del documento" (Blei, Ng y Jordan, 2003: 997). Es decir, que la labor del algoritmo es simplemente presentar una serie de temas, a elección del investigador, que agrupan palabras según su frecuencia en ellos (y ausencia en otros temas).

El software utilizado ha sido Orange Data Mining, bajo Python 3, gracias a su utilidad para trabajar con grandes volúmenes de datos (minería de texto). Este paquete permite múltiples modalidades, como análisis de sentimientos, redes neuronales, topic models, extracción de datos de Twitter, entre otros muchos (Demšar et al., 2013).

Los temas extraídos del análisis LDA se han redefinido bajo la premisa clásica de los encuadres (Entman, 1993). Estos suelen cumplir estas cuatro características: 1) definen problemas; 2) identifican las causas; 3) proponen soluciones; 4) establecen juicios morales. Aunque, en el caso de este estudio se ha decantado por la definición del problema, al ser la Primavera Árabe un acontecimiento de política internacional. Por supuesto, esto no quiere decir que las otras características no estén presentes sino que los fenómenos propios de la política exterior suelen plantearse desde la delimitación de la problemática al ser mucho menos plausible el contacto directo con el fenómeno abordado. En definitiva, se ha optado por estructurar el análisis de forma sencilla pero teóricamente consistente. La operacionalización de encuadres se ha realizado tradicionalmente a partir de la extracción de conglomerados o clústeres. Dicha operacionalización se ha planteado convencionalmente desde técnicas de investigación 
clásicas, como los análisis de contenido y factoriales. La propuesta planteada va un paso más allá al permitir extraer datos estructurados de forma automática y masiva.

Por lo tanto, la pregunta de investigación que este documento plantea es: ¿Cómo ha sido el tratamiento de la prensa española de la realidad árabe durante el periodo post-revueltas 2011? Desde aquí, se intenta responder a esta cuestión desde la conjugación de la comunicación política y la ciencia computacional. El objetivo es realizar una aportación empírica pasados más de nueve años de las movilizaciones. Para ello, el estudio no se centra en un país en concreto sino que pretende arrojar luz sobre qué temas ha tratado la prensa española cuando ha informado de forma general sobre la Primavera Árabe. En definitiva, realizar un análisis de grandes volúmenes de información que permitan realizar inferencias, sin olvidar que el mundo árabe engloba a 22 países con realidades políticas y sociológicas muy diversas.

\section{Resultados}

El desarrollo de las protestas en el mundo árabe ha dejado escenarios muy diversos dependiendo del país que se quiera de analizar. De estos escenarios ha dependido también su inclusión en la agenda mediática, experimentado algunos una extraordinaria cobertura y otros una menor presencia, quedando en algunos casos apariciones testimoniales. En otras palabras, no ha sido lo mismo informar sobre las oleadas de refugiados sirios y su incidencia en Europa que la salida del poder de Omar Al-Bashir en Sudán, país geográfica y culturalmente lejano a España. Esta realidad coincide con el estudio de Árdevol-Abreu (2015), donde afirma que los medios de comunicación privilegian las informaciones sobre política internacional que repercuten sobre los intereses económicos y estratégicos nacionales.

El primer aspecto sobre la relevancia mediática de un suceso político es el número de apariciones de dicho suceso en los medios de comunicación. A este respecto, el gráfico 1 muestra de forma visual la relevancia mediática que ha tenido la Primavera Árabe. Como se mencionó en el anterior apartado, el gráfico no es una representación de la cobertura de países que experimentaron revueltas, en algunos casos muy superior a la que aquí se muestra (como el conflicto sirio), sino a las veces que se mencionó explícitamente el concepto Primavera Árabe.

La cobertura podría dividirse en tres etapas diferenciadas. La primera (2012-2014), es donde se concentra el mayor número de noticias (especialmente en el primer año). Sin embargo, esta concentración no es uniforme ya que se observa un descenso progresivo de noticias, con pequeños repuntes, a medida que los años se desarrollan. Los repuntes podrían hacer referencia a momentos clave en la deriva política de los países en cuestión. Por ejemplo se observa un incremento de noticias en julio de 2013, fecha en la cual fue derrocado Mohammed Morsi en Egipto. Una segunda etapa (2015-2016) marcada por una nueva tendencia ascendente aunque de menor relevancia si la comparamos con el inicio del primer periodo. Durante esta etapa se producen acontecimientos significativos en Europa como la crisis de refugiados o atentados de gran relevancia, como los de París o Bruselas. Y una tercera, etapa (2017-2020) que podríamos catalogar como de descenso estable con pequeñas subidas. Estos pequeños ascensos también coinciden con hechos políticos muy concretos, por citar algunos: enero de 2020, la muerte del sultán de Omán Qabus Bin Said Al-Said o abril de 2019 con la dimisión de Abdelaziz Bouteflika en Argelia. 
Gráfico 1. Evolución de la cobertura sobre la post-Primavera Árabe

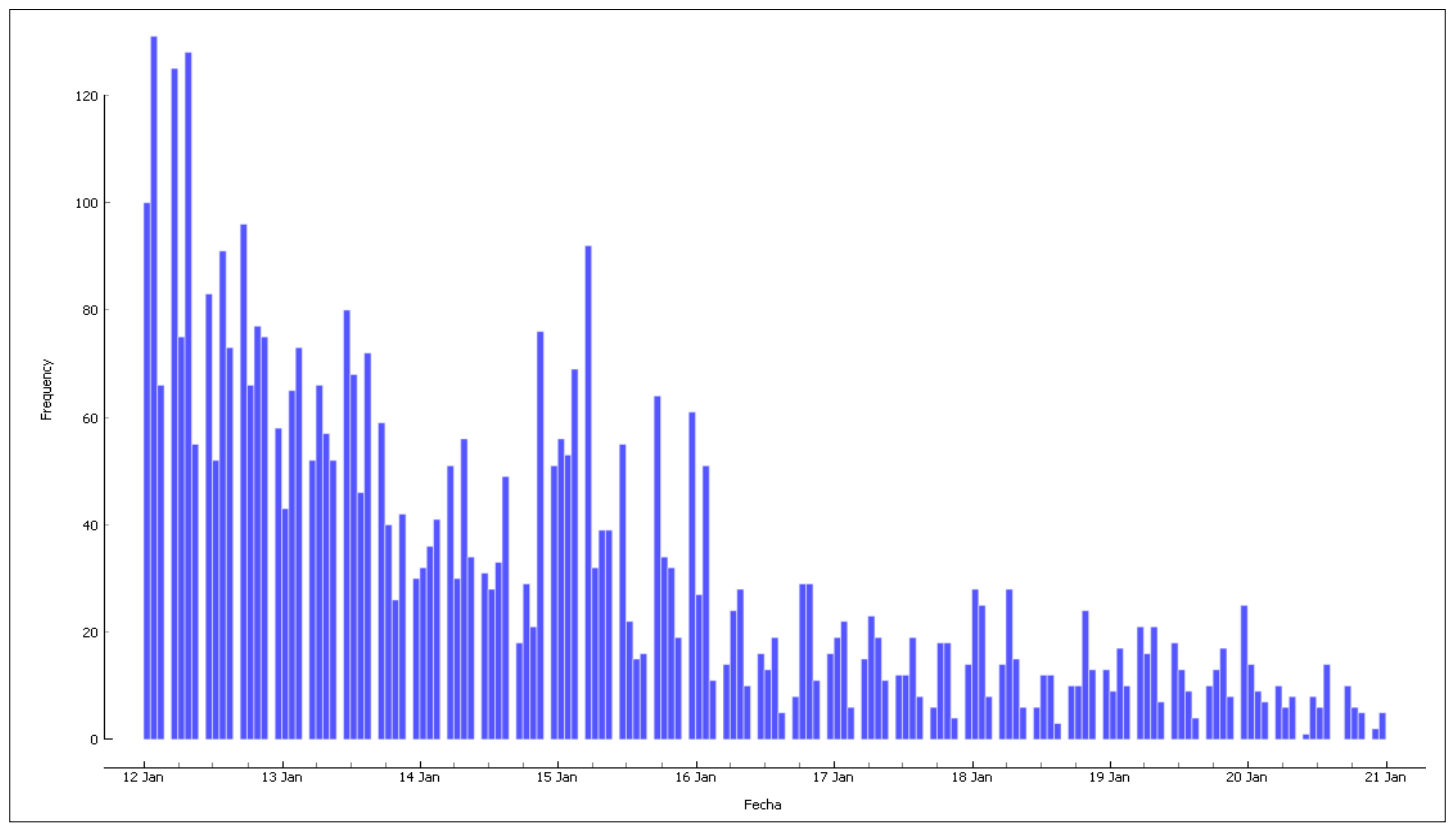

Fuente: elaboración propia

Llegado a este punto surge la pregunta sobre qué países árabes han ocupado mayores titulares en la prensa española. El gráfico 2 y la tabla 1 son bastante clarificadores al respecto a la importancia que cada medio ha dado a los sucesos políticos en el mundo árabe. Los resultados muestran un comportamiento similar sin grandes diferencias entre los medios analizados, lo que sugiere que la razón se encuentra en elementos culturales compartidos. En la tabla se incluyen todos los países árabes (además de Irán) que en algún momento fueron mencionados. Los países árabes que no tuvieron ninguna mención fueron Chad, Comoras y Mauritania. Al igual que en la división de periodos, la presencia de los países en los titulares puede dividirse en grupos bien diferenciados.

En primer lugar, Túnez es el país más mencionado en los medios estudiados (excepto en el caso de El Mundo). Que su presencia sea mayoritaria entra dentro de los resultados esperados ya que fue en el país magrebí donde comenzaron las revueltas que posteriormente se propagaron por la región MENA. Además, los resultados obtenidos invitan a pensar que probablemente el proceso político tunecino, como tema de la agenda mediática, posea unas dinámicas periodísticas específicas. Las posibles explicaciones las podemos encontrar en que Túnez ha sido el único país que ha obtenido puntuaciones positivas en los principales índices de democracia. Igualmente, consideramos que la experiencia tunecina puede presentar elementos altamente noticiables ya que a pesar de haberse producido reformas legales que suponen una mayor garantía en materia de libertades públicas y derechos civiles (Pérez-Beltrán y García-Marín, 2015) la consolidación de la democracia todavía presenta desafíos importantes (Farag, 2020). 
En segundo lugar, se debe destacar la importante cobertura que se dedica al caso de Egipto siendo, tras Túnez y Siria, el tercer país más mencionado. Resulta complejo establecer afirmaciones contundentes al respecto ya que no existe una amplia bibliografía acerca del tema egipcio. A primera vista se puede establecer que el caso egipcio ha sido seguido con gran interés por parte de los medios españoles. Algo lógico si tenemos en cuenta que durante este periodo se produjo el derrocamiento del presidente Mohammed Morsi de los Hermanos Musulmanes. Este interés puede estar relacionado, entre otros motivos, con el acercamiento diplomático español hacia el ejecutivo de El Cairo. De hecho, Azzaola-Piazza y González-González (2017) señalan que los comportamientos de la diplomacia española hacia el gobierno de Abdel Fatah Al-Sisi son muy similares al periodo de Hosni Mubarak.

En tercer lugar, podemos establecer otro bloque de países que han experimentado conflictos armados que perduran en la actualidad. Como se puede observar, Siria es el segundo país que más titulares dedica la prensa. Las diferencias con Yemen son significativas aunque, a nuestro juicio, llama poderosamente la atención los escasos resultados sobre Libia. La explicación más plausible puede ser la dificultad de la prensa de informar sobre dicho conflicto ya que ni el gobierno de España ni la Unión Europea han mostrado una postura unánime hacia el mismo. Además, la cobertura española sobre el conflicto libio ha sido muy desigual viviendo sus momentos de máxima atención mediática con sucesos humanitarios dramáticos (Moreno-Mercado, Luengo y García-Marín, 2021).

Finalmente, en cuarto lugar se encuentran el resto de países presentes en la cobertura. Dentro de estos el que presenta resultados más significativos es Marruecos; algo esperable debido a la cercanía geográfica y a los múltiples intereses españoles en el país. Además, de las potencias regionales que tienen un papel fundamental en el desarrollo político de Oriente Próximo, Arabia Saudí e Irán. El resto de países experimentan un protagonismo menor fuertemente dependiente de hechos políticos de gran transcendencia, como en los casos de Argelia, Bahréin o Irak.

Tabla 1. Frecuencia de países en los titulares por medio

\begin{tabular}{|c|c|c|c|c|c|}
\hline País & ABC & El País & El Mundo & La Vanguardia & Total \\
\hline Túnez & 83 & 89 & 45 & 71 & $\mathbf{2 8 8}$ \\
\hline Siria & 49 & 50 & 60 & 61 & $\mathbf{2 2 0}$ \\
\hline Egipto & 34 & 40 & 46 & 52 & $\mathbf{1 7 2}$ \\
\hline Marruecos & 30 & 29 & 22 & 17 & $\mathbf{9 8}$ \\
\hline Arabia Saudí & 16 & 20 & 16 & 20 & $\mathbf{6 8}$ \\
\hline Irán & 16 & 13 & 18 & 20 & $\mathbf{6 7}$ \\
\hline Yemen & 14 & 16 & 17 & 15 & $\mathbf{6 7}$ \\
\hline Libia & 12 & 19 & 9 & 7 & $\mathbf{5 5}$ \\
\hline Argelia & 5 & 18 & 17 & $\mathbf{4 7}$ \\
\hline Bahréin & 8 & 9 & 15 & $\mathbf{3 9}$ \\
\hline
\end{tabular}




\begin{tabular}{|c|c|c|c|c|c|}
\hline Sáhara Occid & 9 & 4 & 11 & 10 & $\mathbf{3 4}$ \\
\hline Jordania & 10 & 9 & 10 & 4 & $\mathbf{3 1}$ \\
\hline Irak & 11 & 3 & 9 & 6 & $\mathbf{2 7}$ \\
\hline Qatar & 8 & 3 & 6 & 4 & $\mathbf{2 3}$ \\
\hline Kuwait & 3 & 6 & 8 & 4 & $\mathbf{2 1}$ \\
\hline Omán & 6 & 3 & 6 & 4 & $\mathbf{1 9}$ \\
\hline Líbano & 5 & 3 & 5 & 4 & $\mathbf{1 7}$ \\
\hline Palestina & 2 & 5 & 6 & 3 & $\mathbf{1 4}$ \\
\hline EAU & 1 & 6 & 3 & 0 & $\mathbf{1 2}$ \\
\hline Sudán & 4 & 4 & 0 & 1 & $\mathbf{2}$ \\
\hline Yibuti & 2 & 0 & 0 & $\mathbf{1}$ \\
\hline Somalia & 0 & & 1 & 4 & 4 \\
\hline
\end{tabular}

Fuente: elaboración propia

Gráfico 2. Presencia de países seleccionados en los titulares agrupados

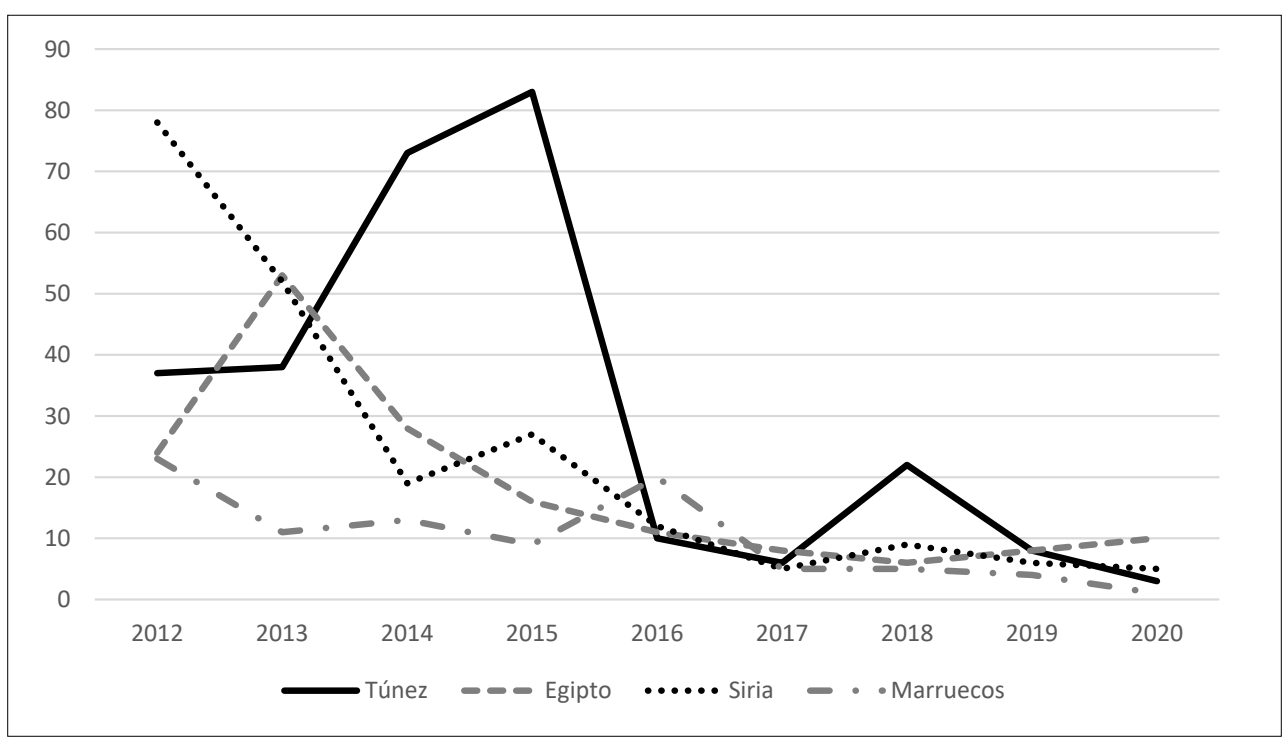

Fuente: elaboración propia 
Se debe señalar que no sólo la cantidad de información y los actores mencionados es suficiente para poder medir cómo ha sido el comportamiento mediático. La manera de informar y los temas tratados supone uno de los elementos más importantes en cualquier análisis sobre cobertura y dimensión informativa. En la tabla 2 se exponen los principales topics-frames extraídos a partir de la aplicación del algoritmo LDA. Los temas se han expuesto a partir de la teoría de encuadres (framing theory), especialmente a partir de la definición del problema, como se mencionó anteriormente en el apartado metodológico. No obstante, queremos destacar que aunque el LDA es una técnica ilustrativa podría ser conveniente combinarse con otras técnicas complementarias, como las máquinas de soporte vectorial (SVM) para localizar encuadres. Una de las características del modelo LDA es que deben aplicarse a textos muy uniformes, lo que implica que hay que tomar cierta prudencia a la hora de establecer causalidades. Por este motivo deben utilizarse de manera exploratoria o descriptiva (objetivo de este trabajo). De la extracción de temas que permite el software Orange (de uno a doce), consideramos que tres es el número más pertinente para este estudio después de descartar otras posibilidades. La razón la encontramos en que la división de la muestra en más temas no arrojaba más resultados que permitiesen aumentar el potencial explicativo de la investigación. Sin embargo, debemos resaltar que, para dar sentido a la investigación, es necesaria la interpretación cualitativa de los autores ya que la aplicación por sí del modelo no es suficiente.

El primero de ellos podríamos definirlo como Conflicto Sirio. El conjunto de términos que lo compone es bastante clarificador en cómo los medios han enfocado la problemática de la crisis siria. Se hace especial hincapié en la brutalidad del régimen en la represión de las protestas y por lo tanto la responsabilidad del presidente Bashar Al-Assad, así como la implicación de actores regionales como Israel e Irán. Los elementos aquí señalados son claramente identificables con encuadres genéricos como Conflicto, Seguridad y Responsabilidad: "Siria, la primera guerra mundial del siglo XXI, El País, 15/03/19", "Asad el monstruo sin dictadura, El Mundo, 12/03/12", “Siria acusa a Israel de bombardear Damasco, La Vanguardia, 05/05/13", "Paramilitares de Irán blindan a Al Assad, $A B C, 03 / 04 / 14$ ".

El segundo tema, Islam, tiene extensas raíces y rutinas profesionales en la prensa española. De hecho, si antes señalábamos que la bibliografía sobre cobertura española y Primavera Árabe es escasa no es así en el caso del islam. Este tema está fuertemente relacionado con el debate si el islam es compatible con el desarrollo democrático de los países árabes, de ahí que Túnez sea un elemento informativo esencial. En este tema las informaciones sobre las elecciones tunecinas y los candidatos de Ennahda ocupan un lugar importante en la agenda mediática: “Túnez elige a una islamista conservadora como alcaldesa, la primera del mundo árabe, $A B C$, 04/07/18”, “Túnez después de la Primavera Árabe, El País, 16/02/17”, “Túnez demuestra que los movimientos islamistas y laicos pueden trabajar juntos, El Mundo, 09/10/15", "La ira islamista se extiende con asaltos violentos en Túnez y Sudán, La Vanguardia, 15/09/12".

El tercer tema se ha catalogado como Inestabilidad regional y hace referencia a los acontecimientos políticos en general en el mundo árabe. Los términos que engloba hacen referencia al caso de diversos países y los diferentes efectos que ha tenido la Primavera Árabe sobre ellos. A diferencia de los otros dos, podríamos decir que es un tema más genérico acerca de la realidad en el mundo árabe: "Los 20 años de Mohammed VI en el trono de Marruecos: de la apertura valiente al estancamiento, El País, 28/07/19", "Las plagas que esperan a Al Sisi en Egipto, El Mundo, 27/05/14", "Detienen al líder opositor en Bahréin, El Mundo, 29/12/13", "La violencia llega a la revuelta del Rif, $A B C$, 17/06/17". 
Tabla 2. Análisis LDA

\begin{tabular}{|c|c|c|c|}
\hline Prioridad & Conflicto Sirio & Islam & Inestabilidad regional \\
\hline 1 & “Siria” & "Árabe" & “Siria” \\
\hline 2 & "Muertos" & "Primavera" & "Año" \\
\hline 3 & "Israel" & "Islam" & "Marruecos" \\
\hline 4 & "Irán" & "Mundo" & "Egipto" \\
\hline 5 & “EEUU” & "Elecciones" & “Nuevo" \\
\hline 6 & “Gobierno” & "Túnez" & "Bahrein" \\
\hline 7 & "Assad" & "Democracia" & “Denuncia” \\
\hline 8 & “Armas" & “Revolución” & "Revuelta" \\
\hline 9 & "Régimen" & "Crisis" & “2011” \\
\hline
\end{tabular}

Fuente: elaboración propia

Por último, se consideró relevante ver si los cuatro medios utilizaron un lenguaje similar o distinto a la hora de construir sus encabezamientos. Para arrojar luz sobre esta cuestión se realizó un análisis simple de frecuencias por medio, materializado en la tabla 3. Los resultados muestran una utilización de lenguaje muy similar, aunque podemos destacar algunas diferencias. Primero, cómo en el caso de La Vanguardia aparecen los términos “turistas" y “Obama”. Estos resultados señalan que los aspectos económicos e internacionales poseen una relevancia significativa: "Los turistas ya pueden hacerse "'”'selfies ".' gratis con las momias de Egipto, La Vanguardia, 01/08/19”, “Obama da un primer paso para encauzar las relaciones con el Golfo Pérsico, La Vanguardia, 15/05/15”. Y, segundo, se destaca la importancia de Arabia Saudí en el caso de El País. Esta importancia se traduce en la relevancia de la Casa de los Saúd en el entorno regional, así como un enfoque crítico con las políticas del país: "Irán mide sus fuerzas con Arabia Saudí en Oriente Próximo, El País, 28/03/15", "Amnistía documenta el acoso a los activistas civiles en Arabia Saudí, El País, 10/10/14".

Tabla 3. 10 términos más utilizados por medio

\begin{tabular}{|c|c|c|c|c|c|c|c|c|c|c|c|}
\hline \multicolumn{3}{|c|}{ ABC } & \multicolumn{3}{c|}{ El País } & \multicolumn{3}{c|}{ El Mundo } & \multicolumn{3}{c|}{ La Vanguardia } \\
\hline Término & $\mathbf{n}$ & $\mathbf{\%}$ & Término & $\mathbf{n}$ & $\mathbf{\%}$ & Término & $\mathbf{n}$ & $\mathbf{\%}$ & Término & $\mathbf{n}$ & $\%$ \\
\hline Árabe & 98 & 8.91 & Túnez & 89 & 9.05 & Siria & 80 & 6.21 & Árabe & 101 & 8.00 \\
\hline Túnez & 83 & 7.55 & Siria & 65 & 7.27 & Árabe & 53 & 4.11 & Siria & 84 & 6.65 \\
\hline Primavera & 76 & 6.91 & Árabe & 56 & 5.69 & Primavera & 50 & 3.88 & Primavera & 72 & 5.70 \\
\hline Siria & 66 & 6.00 & Islam & 56 & 5.69 & Egipto & 45 & 3.49 & Túnez & 71 & 5.62 \\
\hline
\end{tabular}




\begin{tabular}{|c|c|c|c|c|c|c|c|c|c|c|c|}
\hline Nuevo & 43 & 3.91 & Primavera & 44 & 4.47 & Túnez & 45 & 3.49 & Egipto & 52 & 4.12 \\
\hline España & 41 & 3.73 & Egipto & 40 & 4.06 & Muertos & 44 & 3.41 & País & 39 & 3.09 \\
\hline Muertos & 39 & 3.54 & Nuevo & 33 & 3.35 & Egipcio & 38 & 2.95 & Turistas & 36 & 2.85 \\
\hline Años & 35 & 3.18 & Política & 30 & 3.05 & Años & 34 & 2.64 & Años & 34 & 2.69 \\
\hline Egipto & 34 & 3.09 & Saudí & 30 & 3.05 & Islam & 33 & 2.56 & Obama & 34 & 2.69 \\
\hline Marruecos & 30 & 2.72 & Marruecos & 29 & 2.95 & España & 33 & 2.56 & Mundo & 33 & 2.61 \\
\hline
\end{tabular}

Fuente: elaboración propia

\section{Discusión y conclusiones}

Esta investigación ha tenido dos objetivos claramente diferenciados pero compatibles: por un lado, aportar que los nuevos análisis cuantitativos son útiles para, al menos, describir la realidad que nos rodea. Aunque, volvemos a resaltar la necesidad de que los autores encuentren un sentido explicativo a los datos extraídos del procesamiento automático. Y, por otro lado, que los medios de comunicación suelen comportarse de acuerdo a referentes culturales compartidos cuando no hay diferencias ideológicas subyacentes (una de las tesis de la teoría del indexado propuesta por Lance Bennett, véase, por ejemplo, su definición de 2015). La combinación de ambos nos ha servido para mostrar, a grandes rasgos, la cobertura de los medios españoles seleccionados sobre la Primavera Árabe (cualquiera que sea el significado del término) durante ocho años. A este respecto, el análisis no arroja grandes sorpresas: los medios analizados cubrieron en más ocasiones y de forma más profunda aquellos sucesos llamativos y culturalmente cercanos. Datos que nos invitan a pensar que los principios del gatekeeping siguen igual de vigentes ahora que hace 70 años cuando se propusieron. Una ausencia de sorpresas que puede constituir una llamada de atención. Y, es que, la información que reciben los ciudadanos, incluso en esta época de sobreabundancia informativa, sigue rigiéndose por patrones que limitan su alcance: sigue habiendo lagunas, estereotipos y, en este caso al menos, una ausencia patente de pluralismo. Si bien, estas conclusiones pueden deberse a la elección concreta de la muestra escogida consideramos que es un elemento que debe invitar a la reflexión académica en futuras investigaciones.

Efectivamente, las informaciones que los medios seleccionados han elaborado pueden caracterizarse por ser muy similares, hasta el punto de que con sólo tres agrupaciones de términos (elaborados por el algoritmo) podemos resumir la cobertura durante ocho años de cuatro diarios en apariencia diversos aunque con singularidades comunes. Realmente, hay países que apenas son mencionados, incluso cuando los ejemplos de cambio político son recurrentes y la reacción gubernamental muy violenta, como es el caso de Bahréin. Son datos que invitan a la reflexión sobre la calidad y variedad de las informaciones de que disponen los ciudadanos y de los prejuicios y juicios de valor a que pudieran dar lugar. En este sentido, podemos establecer que esta investigación pretende arrojar luz sobre un objeto de estudio que ha contado con más afirmaciones que demostraciones. De hecho, puede encuadrarse dentro de la reflexión general de hacia donde camina el periodismo internacional en el complejo mediático español. En conclusión, este trabajo puede ayudar al debate científico acerca las lecciones aprendidas y los retos que plantean las relaciones de España con el mundo árabe tras las revueltas del 2011. Debate que sigue muy vigente dentro de la academia 
española como lo demuestran obras y encuentros científicos de reciente elaboración, como Movilizaciones populares tras las Primaveras Árabes (2011-2021).

Igualmente, como hemos mencionado, una de las aportaciones que pretendemos hacer con esta investigación es metodológica. En la actualidad el número de medios de comunicación se ha multiplicado y los canales por los que emiten son digitales. Esto hace que sea información no estructurada, texto, pero a disposición de los investigadores. Véase la capacidad de extraer grandes volúmenes de datos de medios digitales (través de MyNews o Lexis UNI) y redes sociales, como Twitter (API REST) pero que necesitan ser tratados y categorizados. Nunca antes han dispuesto los académicos de tanta información ni de tanta capacidad de análisis a un precio irrisorio. Por ello, las técnicas metodológicas deben forzosamente adaptarse a un nuevo entorno tan variado en el que los análisis sobre un medio o canal cada vez es menos significativo. Es necesario ampliar el conjunto de datos para tener una imagen certera de la realidad informativa (o social). Por supuesto, esta innovación metodológica ha ido acompañada del desarrollo tecnológico en los últimos años. Por lo que podemos decir que este trabajo utiliza una metodología actual en un campo de investigación (comunicación internacional) que todavía adolece de estudios cuantitativos ambiciosos. Lo que aquí proponemos no es más que una técnica exploratoria, descriptiva, pero válida para hacer ese tipo de trabajo cada vez más común entre la comunidad científica. Los algoritmos de clasificación y análisis (supervisados o no) y la mal llamada inteligencia artificial deben convertirse en herramientas habituales de los investigadores en ciencias sociales. Sólo así seremos capaces de acercarnos a unas realidades cada vez más fragmentadas y digitales.

\section{Agradecimientos}

Este artículo ha sido traducido al inglés por Laura Aitken.

\section{Referencias bibliográficas}

Abu-Tarbush, J. (2015). Estados Unidos y la promoción de la democracia en Oriente Medio y el Norte de África. En Álvarez-Ossorio, I. (ed.), La Primavera Árabe revisitada (pp. 25-50). Navarra: Aranzadi.

Altouragh, A.F., Chen, K.W.y Al-Kandari, A.A. (2021). The Relationship between Media use, perceptions and regime preference in post-Arab Spring countries. Global Media and Communication, 1-29. https://doi.org/10.1177/17427665211001894

Álvarez-Ossorio, I., Barreñada-Bajo, I. y Mijares-Molina, L. (2021). Movilizaciones populares, regresión autoritaria y horizonte postpandemia en el Magreb y Oriente Próximo. En Álvarez-Ossorio, I., Barreñada-Bajo, I. y Mijares-Molina, L. (coord.), Movilizaciones populares tras las Primaveras Árabes (2011-2021) (pp. 11-32). Madrid: La Catarata.

Ardèvol-Abreu, A. (2015). Construcción de la agenda y de los encuadres noticiosos de los países en situación de crisis humanitaria: valores-noticia y selección de fuentes. Communication \& Society, 28(1), 43-62. doi: 10.15581/003.28.1.sp.43-62

Atlas, P.M. (2012). U.S. Foreign Policy and the Arab Spring: Balancing Values and Interests. Digest of Middle East Studies, 21(2), 353-385. https://doi.org/10.1111/j.1949-3606.2012.00158.x 
Azzaola-Piazza, B. y González-González, I. (2017). Las relaciones de España y el Egipto post-Mubarak (2011-2015). Revista de Estudios Internacionales Mediterráneos (REIM), (23), 29-46. https://doi.org/10.15366/reim2017.23.003

Blei, D., Ng, A. y Jordan, M. (2003). Latent Dirichlet Allocation. Journal of Machine Learning Research, (3), 993-1022.

Bennett, L. (2015). Indexing theory. En, Mazzoleni, G., Barnhust, K., Ikeda, K., Maia, R. y Wessler, H. (eds.), The International Encyclopedia of Political Communication (pp.1-5). Wiley.

Corral-García, A. y Fernández-Romero, C. (2015). "Framing” y mundo árabe: la cobertura de la prensa española en torno a la revolución tunecina”. Estudios sobre el Mensaje Periodístico, 21(2), 793-811.https://doi.org/10.5209/rev_ESMP.2015.v21.n2.50885 Corral, A, Fernández-Romero, C. y García-Ortega, C. (2020). Framing e Islamofobia. La cobertura de la revolución egipcia en la prensa española de referencia (2011-2013). Revista Latina de Comunicación Social, (77), 373-392. https://doi.org/10.4185/ RLCS-2020-1463

Dastgeer, S., y Gade, P., (2016). Visual Framing of Muslim Women in the Arab Spring: Prominent, Active and Visible. International Communication Gazzette, 78(5), 432-450. https://doi.org/10.1177/1748048516640204

Demšar, J,, Curk, T., Erjavec, A., Gorup, C., Ho evar, T., Milutinovi , M., Možina, M., Polajnar, M., Toplak, M., Stari , A., Štajdohar, M., Umek, L., Žagar, L., Žbontar, J., Žitnik, M. y Zupan, B. (2013). Orange: Data Mining Toolbox in Phyton. The Journal of Machine Learning Research, 14(1), 2349-2353.

Durán-Cenit M. y García-Marín, J. (2015). Libertad de expresión y regulación mediática en la Turquía de Erdogán. En Álvarez-Ossorio, I. (ed.), La Primavera Árabe revisitada (pp. 191-210). Navarra: Aranzadi.

Elena, M. y Tulloch, C. (2017). Los Social Media como fuente de información en conflictos internacionales. Fuentes y praxis periodística en los informativos de la televisión de Cataluña durante la Primavera Árabe egipcia. Hipertext.net: Revista Académica sobre Documentación Digital y Comunicación Interactiva, (15), 46-56.

El-Hadadi, A. (2020). Redefining the Social Contract in the Wake of the Arab Spring: The Experiences of Egypt, Morocco and Tunisia. World Development, 127, 1-22. DOI: 10.1016/j.worlddev.2019.104774

Engineer, A. (2008). The Rights of Women in Islam. Nueva Delhi: Sterling Publishers.

Entman, R. (1993). Framing: A Toward Clarification of a Fractured Paradigm. Journal of Communication, 43(4), 51-58. https://doi. org/10.1111/j.1460-2466.1993.tb01304.x

Farag, M. (2020). Mass-Elite Differences in New Democracies: Tunisia as a Case of Study. European Political Science, 19(4), 550561. https://doi.org/10.1057/s41304-020-00274-x

Freedom House (2020). Freedom in the World 2020: A Leaderless Struggle for Democracy", Freedom House. https://freedomhouse.org/sites/default/files/2020-02/FIW_2020_REPORT_BOOKLET_Final.pdf (Consultado el 20 de diciembre de 2020).

García-Marín, J. (2017). Media and Media Freedom. En Szmolka, I. (ed.), Political Change in the Middle East and North Africa: After the Arab Spring (pp. 231-256). Edinburgo: Edinburgh University Press.

Hovy, D. (2020). Text Analysis in Python for Social Scientists. Cambridge: Cambridge Elements. 
Irakrak, N. (2017). El tratamiento de la revuelta árabe en la prensa española y marroquí (2010-2011), Sevilla: Universidad de Sevilla.

Luengo, Ó. y Fernández-García, B. (2017). Political Participation and New TecÝologies of Communication in Spain. Przegl $d$ Politologiczny 3, 21-31.

Martínez-Fuentes, G. (2011). Redes sociales y mundo árabe. Lenguaje y significado. Telos: Cuadernos de comunicación e innovación, (89), 105-107.

Meraz, S. y Papacharissi, Z. (2013). Networked Gatekeeping and Networked Framing on \#Egypt, The International Journal of Press/Politics, 18(2), 138-166. https://doi.org/10.1177/1940161212474472

Moldovan, R. (2020). The Social Media Revolution That Failed: Lessons From Arab Spring. Redefining Community in Intercultural Context, 9(1), 257-269.

Moreno-Mercado, J.M (2018). La cobertura mediática de los conflictos armados. El caso de Siria. Revista de Paz y Conflictos, 11(2), 115-142. https://doi.org/10.30827/revpaz.v11i2.7585

Moreno-Mercado, J.M., Luengo, Ó., y García Marín J. (2021). Cyberspace as a Global Common: Framing the Lybian War in RT, RTVE and La Sexta Television Videos. En Martín Ramírez J. y Bauzá Abril, B. (coord.), Security and the Global Commons and Beyond (pp. 129-142). Cham: Springer.

Omar Alsalem, Z. (2016). La imagen de Arabia Saudí en la prensa española: un análisis del discurso de ABC, El Mundo y El País. Madrid: Universidad Autónoma de Madrid.

Orosa, B., Santorum, S. y García, X. (2017). El uso del clickbait en cibermedios de los 28 países de la Unión Europea. Revista Latina de Comunicación Social, 72, 1261-1277. DOI: 10.4185/RLCS-2017-1218

Ostrowiski, D.A. (2015). Using Latent Dirichlet Allocation for Topic Modelling in Twitter. En Proceedings of the 2015 IEEE $9^{\text {th }}$ International Conference on Semantic Computing (pp. 493-497). IEEE.

Pérez-Beltrán, C. y García-Marín, J. (2015). Las libertades públicas en Túnez tras las revueltas del 2011. Revista CIDOB d'afers internaticionals (109), 69-90.

Plaza, J., Rivas-Nieto, P. y Rey-García, P. (2017). La representación de las mujeres terroristas y víctimas del terrorismo en prensa española. El caso de El País". Revista Latina de Comunicación Social, (72), 129-144. DOI: 10.4185/RLCS-2017-1157

Priego-Moreno, A. (2011). La Primavera Árabe: ¿Una cuarta ola de democratización? UNISCI Discussion Papers, (26), 75-94.

Sánchez-Castillo, S. y Zarauza-Valero, T. (2020). El desplazamiento de los refugiados ante las cámaras de los informativos de TVE. Tonos Digital, 38(0), 1-26.

Sanz-Ocaña, F. (2018). La construcción del framing en el caso de las Primaveras Árabes en la prensa de referencia española. Valladolid: Universidad de Valladolid

Sarnelli, V. (2013). Tunisia, Egypt and the Voices of the Revolution in Al Jazeera English. Journal of Arab \& Muslim Media Research, 6(2-3), 157-176. DOI: 10.1386/jammr.6.2-3.157_1 
Sik Ha, J. y Shin, D. (2019). Portraying China as an Alternative to U.S. Hegemony: The China Daily's Framing of the Arab Spring. Atlantic Journal of Communication, 27(3), 200-215. https://doi.org/10.1080/15456870.2019.1610764

Soria-Ibáñez, M.M. (2013). El tratamiento informativo de la Primavera Árabe: Libia y su papel en las portadas españolas. Encuentros, 11(1), 25-39.

Szmolka, I. (2013). ¿La quinta ola de democratización?: Cambio político sin cambio de régimen en los países árabes. Política y Sociedad, 50(3), 893-935. https://doi.org/10.5209/rev_POSO.2013.v50.n3.41350

The Economist. (2019). Democracy Index 2019. The Economist Intelligence Unit, 2019. https://www.eiu.com/topic/democracy-index (Consultado el 20 de diciembre de 2020).

Truevtsev, K. (2020). 2019 Turn: The New Stage of the Arab Spring or Start of The New Paradigm of Political Development? Vostok. Afro-Aziatskie Obshchestva: Istoriia i Sovremennost, (2), 96-105.

Van Dijk, T. (1990). Social Cognition and Discourse. En Giles, H y Robinson, P. (eds.), The New Handbook of Language and Social Psychology (pp. 163-183). Chichester: Wiley.

Vidal-Valiña, C.M. (2013). Las revueltas de Libia de 2011 a través de la mirada de los periodistas de TVE: ¿Un cambio de paradigma sobre la cobertura de la región MENA? En Fernandez-Rodríguez, M., Bravo-Díaz, D. y Martínez-Peñas, L. (coord..). Una década de cambios: de la guerra de Irak a la evolución de la Primavera Árabe (2003-2013) (pp. 183-201). Madrid: Asociación Veritas para el Estudio de la Historia, el Derecho y las Instituciones.

Wilson, C. y Dunn, A. (2011). Digital Media in the Egyptian Revolution: Descriptive Analysis from the Tahrir Data Sets. International Journal of Communication, 5, 1248-1272. 